\title{
Effects of Menstrual Phase on Intake of Nicotine, Caffeine, and Alcohol and Nonprescribed Drugs in Women With Late Luteal Phase Dysphoric Disorder
}

\author{
Judith L. Marks \\ University of Michigan \\ Catherine S. Hair \\ University of Connecticut \\ Susan C. Klock \\ Harvard University \\ Benson E. Ginsburg \\ University of Connecticut \\ Cynthia S. Pomerleau \\ University of Michigan
}

\begin{abstract}
To investigate the possibility that cigarette smoking and other drug use are affected by menstrual phase in smokers with Late Luteal Phase Dysphoric Disorder (LLPDD), we examined daily diaries rating menstrual symptomatology, smoking, alcohol and nonprescription drug use, and caffeine intake in nine female smokers meeting criteria for LLPDD. Menstrual symptomatology peaked during the premenstrual phase. Smoking, alcohol, and nonprescription drug intake were increased during menses; caffeine intake was unaffected by phase. No systematic intrasubject correlation between symptomatology and smoking was detected. It was concluded that in women with LLPDD, smoking and alcohol and nonprescription drug intake appear to vary as a function of menstrual phase. The lack of intrasubject correlations between symptomatology and intake, and the failure of peak intake to coincide with peak symptomatology, however, indicate that these effects cannot be explained simply as "self-medication" of acute episodes of dysphoric mood.
\end{abstract}

Data were collected by the first author as part of her doctoral dissertation at the University of Connecticut; the work was supported by a grant from the University of Connecticut Foundation to the fourth author and a grant from the University of Connecticut Health Center to the second author. Preparation of the article was supported Grant CA 42730 to Ovide F. Pomerleau by the National Cancer Institute. The authors wish to thank Nancy Reame for her helpful comments on an earlier draft of this manuscript.

Correspondence and requests for reprints should be sent to Judith L. Marks, University of Michigan, Department of Neurology, Mental Health Research Institute, 205 Zina Pitcher Drive, Ann Arbor, MI 48109-0720. 
Although a number of investigators have attempted to determine whether menstrual cycle phase affects intake of a number of drugs, including caffeine (Schechter, Bachmann, Vaitukaitis, Phillips, \& Saperstein, 1989), alcohol (Mello, 1986; Schechter et al., 1989), marijuana (Griffin, Mendelson, Mello, \& Lex, 1986), and nicotine (Mello, Mendelson, \& Palmieri, 1987; Pomerleau, Cole, Lumley, Marks, \& Pomerleau, 1994; Pomerleau, Garcia, Pomerleau, \& Cameron, 1992; Steinberg \& Cherek, 1989), the weight of evidence for the most part favors the absence of phase differences in substance use.

An exception to this generalization is provided by a number of studies documenting menstrual phase differences in the intake of alcohol (Belfer, Shader, Carroll, \& Hermatz, 1971; Podolsky, 1963) and marijuana (Mello, 1986) in women with premenstrual dysphoria. Intake peaked during the premenstruum, leading Mello (1986) to suggest that the depression and anxiety associated with the premenstruum in these women provoked episodic increases in drug use. In an effort to determine whether smoking also varies as a function of menstrual phase in female smokers with premenstrual symptomatology, whether other drug use also showed phase differences in these smokers, and whether increased substance use was associated with either affective disturbances or physical discomfort, we examined data from nine female smokers meeting criteria for Late Luteal Phase Dysphoric Disorder (LLPDD) who were asked to keep daily diaries rating menstrual symptomatology, smoking, and other substance intake.

\section{METHOD}

\section{Subjects}

Subjects were nine female smokers who participated in a larger study designed to determine what percentage of women perceiving themselves as having premenstrual syndrome actually meet criteria for LLPDD as proposed in the $D S M-I I I-R$ (American Psychiatric Association, 1987) and whether those who met criteria had different family histories of psychopathology than those who did not (Marks, 1993; Marks, Hair, Klock, \& Ginsburg, under review). Subjects were recruited from the local community using advertisements in a local newspaper and a hospital employee newsletter seeking women who perceived themselves as suffering from premenstrual syndrome to participate in a study of phase-related mood change. They were selected without regard to smoking status or smoking history. To be eligible, candidates were required: (a) to be between the ages of 18 and 45; (b) to be in good health; (c) to not be taking any psychoactive medication or hormones, including oral contraceptives, currently or in the past 3 months; (d) to not be pregnant currently or within the past 6 months, or planning a pregnancy during the course of the study; and (e) to have regular menstrual cycles.

Following telephone screening, 75 women appeared to be eligible for the study and were scheduled for an initial interview. Twenty women canceled their appointments, 5 were disqualified for failure to meet study criteria, and 25 withdrew after enrollment. Of the 25 women who completed the study, 19 ( 9 smokers, 3 ex-smokers, 7 lifetime nonsmokers) met criteria for LLPDD; the 9 smokers served as subjects in the present study. Subjects were paid for their participation in the study. 
Subjects had a mean age of $31.8(S D \pm 5.3)$ years and reported smoking a mean of $18.8(S D \pm 4.9)$ cigarettes per day. All but two subjects (one with a history of depression, one with a current diagnosis of social phobia and a history of simple phobia and panic disorder) were free of comorbid diagnoses. All but one subject were high school graduates, and six of the nine had at least some college education. All subjects were employed-one in an unskilled job, one in a semiskilled job, with the remainder professionally employed. Three subjects were married, three were divorced, and three were single; two had one or more children. All subjects were white.

\section{Procedure}

At the initial interview, the study was explained, informed consent obtained, and premenstrual symptomatology reviewed. The subject then completed the Premenstrual Assessment Form (Halbreich, Endicott, Schacht, \& Nee, 1982), a retrospective self-report questionnaire containing 95 items querying premenstrual changes and collecting information about demographics, health, and menstrual cycle phenomena.

The study required subjects to complete the 21-item Daily Ratings Form (DRF; Endicott \& Halbreich, 1982; Endicott, Nee, Cohen, \& Halbreich, 1986) for at least two consecutive menstrual cycles. The DRF prospectively assesses symptoms along five dimensions (dysphoric mood, physical discomfort, low energy, more alcohol/sex/activity, and consumption). Substance intake is assessed by items querying smoking, intake of alcohol and nonprescribed drugs (combined in a single item), and intake of caffeinated beverages. In this study, three items (difficulty concentrating, decreased appetite, insomnia) were added to facilitate diagnosis of LLPDD. All 24 items were rated on a 6-point scale ranging from "not at all" to "extreme." Subjects were instructed to fill out the DRF at bedtime. Forms were mailed to the experimenter following each cycle using preaddressed, postage-paid envelopes.

Subjects were diagnosed as having LLPDD if their DRF ratings met at least one of two sets of criteria: (a) The method of Rivera-Tovar and Frank (1990) requires a $30 \%$ increase above baseline during the 7 days prior to menses, compared with the 7 days after onset of menses, for at least 5 of the 10 symptoms listed in the Criterion B of the $D S M-I I I-R$ criteria, over at least two menstrual cycles; average daily postmenstrual rating for these symptoms must be $<3$; (b) The "absolute severity criteria" of Eckerd, Hurt, and Severino (1989; Hurt et al., 1992) require that 5 of the $10 D S M-I I I-R$ symptoms have at least one "severe" $(\geq 5)$ rating during the 6 days prior to menses and that ratings for at least 4 of 6 days after menses be not greater than "minimal" $(\leq 2)$, for at least half of the recorded cycles. Both sets of guidelines require that symptoms meeting criteria must include one of the following: affective lability (mood swings), anger/ irritability, anxiety, or depressed mood. Data were collected for at least two complete cycles in all subjects.

After submitting her last DRF, each subject attended a final session in which history of psychopathology was assessed using a self-administered, computerinteractive version of the National Institute of Mental Health Diagnostic Inter- 
view Schedule-III-R (C-DIS; Blouin, 1991). Both the initial and final interviews were conducted 1 to 7 days post menses.

\section{Analysis of Menstrual Cycle Data}

The statistical and conceptual problems involved in analyzing menstrual cycle data are widely recognized (Steege, 1989); even the most sophisticated methodologies have shortcomings. Our approach, based on the Society for Menstrual Cycle Research guidelines (Sommer, 1986), involved calculating means for five phases defined as follows: menses (all bleeding days), premenstrual ( -7 through -1 days before the onset of bleeding), ovulatory ( -16 through -12 days before onset of bleeding), postovulatory ( -11 through -8 days before the onset of bleeding), and postmenses (the end of menses to the beginning of the ovulatory phase). 1 Means were then compared using repeated-measures analyses of variance (ANOVAs); a posteriori analyses were conducted using the Newman-Keuls test (Duncan, 1957; Winer, 1971). Although a few subjects provided data for three cycles, only data from the first two cycles were included to ensure comparability between cycles; because preliminary analyses produced no cycle or cycle-byphase effects for any variable, data were pooled across cycles.

Because our hypotheses related primarily to phasic changes in mood or physical state, and because two of the dependent variables (alcohol and nonprescribed drugs; caffeine) were embedded in the more alcohol/sex/activity and consumption dimensions, we restricted our analyses of menstrual symptomatology to the dysphoric mood and physical discomfort dimensions. The dysphoric mood dimension included the following items: mood swings; irritable, angry, impatient; depressed, sad, low, blue, lonely; anxious, jittery, nervous; and stay home, avoid social activity. Items comprising the physical discomfort dimension were abdominal pain; breast pain; less sexual interest; back, joint, or muscle pain; and feel bloated, have edema. Phase means for the DRF items querying smoking, intake of alcohol and nonprescribed drugs, and intake of caffeinated beverages were also analyzed. Pearson intrasubject correlations were calculated for each subject to determine the association between each day's smoking and symptom ratings, independent of phase.

\section{RESULTS}

Not surprisingly (because some of the symptoms contributing to this score formed the basis of diagnosis), significant phase effects were detected for the dysphoric mood dimension (Figure 1, top panel; $F(4,32)=6.31, p<.001$ ), with self-ratings being high during menses, lowest during postmenses, then rising to a peak during the premenstrual phase. Post hoc analyses showed that premenstrual ratings significantly exceeded ratings for menses $(p<.05)$, postmenses $(p$ $<.01$ ), and the ovulatory phase $(p<.01)$ and marginally exceeded the post-

'These terms are preferred to follicular and luteal, which the guidelines suggest be reserved for phase designations based on direct biological measures. 

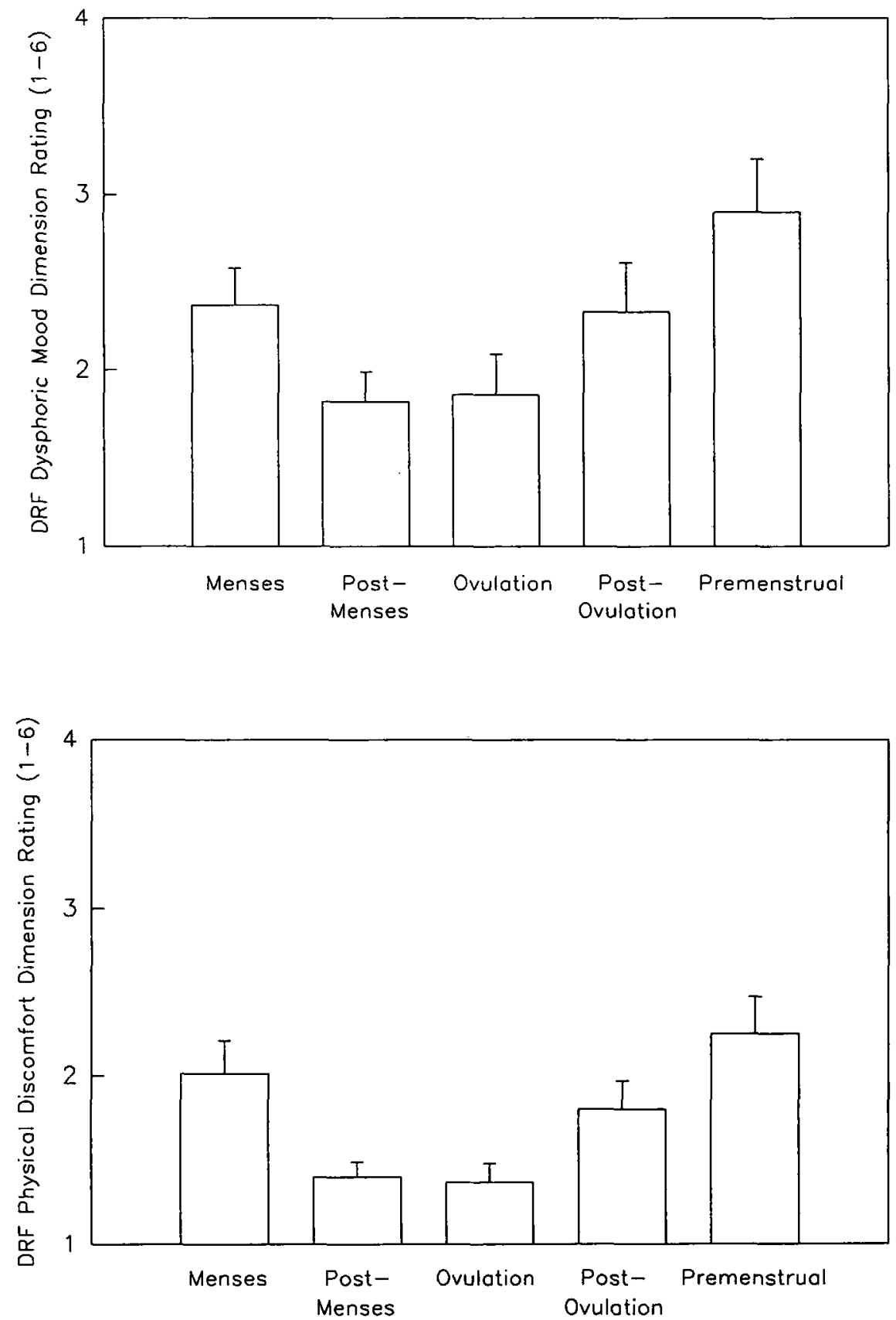

Figure 1. Top panel: Daily self-rating of DRF Dysphoric Mood Dimension (mean for each phase collapsed across two cycles; score represents a mean of five items scored on a scale of 1 (not at all) to 6 (extreme). Bottom panel: Daily self-rating of DRF Physical Discomfort Dimension (mean for each phase collapsed across two cycles; score represents a mean of five items (Mean \pm SEM; $N=9$ ). 



Figure 2. Top panel: Daily self-rating of DRF "Smoke" item (mean for each phase collapsed across two cycles); items are scored on a scale of 1 (not at all) to 6 (extreme). Center panel: Daily self-rating of DRF "Drink alcohol/use nonprescribed drugs" item (mean for each phase collapsed across two cycles). Bottom panel: Daily self-rating of DRF "Drink coffee" item (mean for each phase collapsed across two cycles) (Mean \pm SEM; $\boldsymbol{N}=9$ ). 
ovulatory phase $(p<.10)$. Postovulatory phase ratings marginally exceeded ratings for the ovulatory phase $(p<.10)$. Significant phase effects were likewise observed for the physical discomfort dimension (bottom panel; $F(4,32)=12.49$, $p<.001$ ), with ratings reflecting a pattern similar to that for the dysphoric mood dimension. Premenstrual ratings significantly exceeded those for postmenses $(p$ $<.01)$, the ovulatory phase $(p<.01)$, and the postovulatory phase $(p<.05)$. Ratings for the menstrual phase were significantly higher than for the postmenses $(p<.01)$ and the ovulatory phase $(p<.01)$. Postovulatory ratings significantly exceeded ratings for the postmenstrual $(p<.05)$ and ovulatory $(p<.05)$ phases.

As shown in Figure 2 (top panel), ratings of amount of smoking varied significantly across menstrual phase $(F(4,32)=3.41, p<.05)$, with peak intake occurring during the menses. Menstrual phase ratings significantly exceeded postovulatory ratings $(p<.01)$ and marginally exceeded ratings for both the postmenstrual $(p<.10)$ and premenstrual phases $(p<.10)$. Ratings of intake of alcohol and nonprescribed drugs also varied significantly across menstrual phase (center panel; $F(4,32)=3.84, p<.05$ ), again peaking during the menses. Ratings during menses significantly exceeded those during all other phases (postmenses, $p<.05$; ovulatory, $p<.01$; postovulatory, $p<.05$; and premenstruum, $p$ $<.05$ ). Ratings of caffeine intake (bottom panel), by contrast, did not vary across phase $(F(4,32)=0.23$, n.s. $)$.

For the dysphoric mood dimension, mean intrasubject correlations with smoking were $r=+.17$ during the first month and $r=+.19$ for the second month; corresponding means for the physical discomfort dimension were $r=$ +.06 and $r=+.05$. No more than three subjects showed a significant correlation for either scale for either month.

\section{DISCUSSION}

Significant menstrual phase effects were observed for both physical and affective symptomatology, with both peaking in the premenstruum (though mean levels were not remarkably high for any phase). Subjects also showed significant menstrual phase differences in smoking. Although there was some suggestion of the predicted increase during the premenstruum, the only significant elevation occurred during the menses. A similar pattern emerged for alcohol and nonprescribed drugs, with the association of increased consumption with the menses being even more pronounced.

The failure of peak smoking ratings to correspond with peak symptomatology, combined with the unimpressive mean intrasubject correlations of symptomatology with smoking, suggests that the explanation for phase differences in smoking lies elsewhere (e.g., a phase lag in response, or a greater tendency of women with LLPDD to increase smoking in response to dysmenorrhea) than in the symptoms examined.

The lumping of alcohol with unspecified nonprescription drugs in the DRF complicates interpretation of this variable. Because cigarette smoking often accompanies marijuana as well as alcohol use (Mello, Mendelson, Sellers, \& 
Kuehnle, 1980), it is possible that changes in smoking influenced use of either or both drugs (or vice versa). Likewise, it is impossible to dismiss the possibility that a similar pattern for this variable reflects an increase in use of over-the-counter painkillers during menses. Thus, our study cannot be interpreted as a disconfirmation of previous findings of peak intake of alcohol and marijuana during the premenstruum in women with premenstrual dysphoria (Belfer et al., 1971; Mello, 1986; Podolsky, 1963). Failure to detect phase-related changes in caffeine consumption suggests that even if the underlying mechanisms for phase effects on substance intake in women with LLPDD cut across drugs, they do not necessarily extend to all drugs.

Several additional methodological limitations must be mentioned. The use of ratings collected once at the end of the day, using a relational Likert-type scale rather than quantitative data, constitutes a highly imprecise reflection of smoking rate and drug intake. Because of these limitations, the actual magnitude of the differences in intake cannot be determined and may be quite small in clinical terms. It must also be noted that subjects were not blind to our interest in the menstrual cycle and may have been influenced by expectancies; given the focus on LLPDD, however, such expectancies should have been reflected by maximum ratings during the premenstrual phase, when in fact the highest ratings were observed during menses. Finally, because diaries were collected only at the end of each cycle, we cannot be sure that subjects actually completed them on a daily basis, undermining the prospective nature of the study.

Because of growing evidence of a connection between smoking and a number of psychopathological conditions, including depression (Glassman, 1993; Glassman et al., 1990; Kendler et al., 1993), alcoholism (Dreher \& Fraser, 1967; Istvan \& Matarazzo, 1984), and eating disorders (Pomerleau \& Krahn, 1993), many clinical researchers believe that success in treating smoking in individuals with these conditions will be greatly enhanced by the development of properly timed pharmacological or behavioral interventions that address these co-factors as well. If our findings can be replicated in a larger number of subjects, using more precise measures of nicotine intake, they may likewise help us to recognize and address the special needs of smokers with LLPDD.

\section{REFERENCES}

American Psychiatric Association. (1987). Late luteal phase dysphoric disorder. In Diagnostic and statistical manual of mental disorders (3rd ed., rev., pp. 367-369). Washington, DC: Author.

Belfer, M.L., Shader, R.I., Carroll, M., \& Hermatz, J.S. (1971). Alcoholism in women. Archives of General Psychiatry, 25, 540-544.

Blouin, A. (1991). C-DIS operations manual. Ottawa, Canada: C-DIS Management Group.

Dreher, K.F., \& Fraser, J.G. (1967). Smoking habits of alcoholic outpatients. I. International Journal of Addictions, 2, 259-270.

Duncan, D.B. (1957). Multiple range tests for correlated and heteroscedastic means. Biometrics, 20, $482-491$.

Eckerd, M.B., Hurt, S.W., \& Severino, S.K. (1989). Late luteal phase dysphoric disorder: Relationship to personality disorders. Journal of Personality Disorders, 3, 519-529.

Endicott, J., \& Halbreich, U. (1982). Psychobiology of premenstrual change. Psychopharmacological Bulletin, 18, 109-112. 
Endicott, J., Nee, J., Cohen, J., \& Halbreich, U. (1986). Premenstrual changes: Patterns and correlates of daily ratings. Journal of Affective Disorders, 10, 127-135.

Glassman, A.H. (1993). Cigarette smoking: Implications for psychiatric illness. American Journal of Psychiatry, 150, 546-553.

Glassman, A.H., Helzer, J.E., Covey, L.S., Cottler, L.B., Stetner, F., Tipp. J.E., \& Johnson, J. (1990). Smoking, smoking cessation, and major depression. Journal of the American Medical Association, $264,637-640$.

Griffin, M.L., Mendelson, J.H., Mello, N.K., \& Lex, B.W. (1986). Marijuana uses across the menstrual cycle. Drug and Alcohol Dependence, 18, 213-224.

Halbreich, U., Endicott, J., Schacht, S., \&c Nee, J. (1982). The diversity of premenstrual changes as reflected in the Premenstrual Assessment Form. Acta Psychiatrica Scandinavica, 65, 46-65.

Hurt, S.W., Schnurr, P.P., Severino, S.K., Freeman, E.W., Gise, L.H., Rivera-Tovar, A., \& Steege, J.F. (1992). Late luteal phase dysphoric disorder in 670 women evaluated for premenstrual complaints. American Joumal of Psychiatry, 149, 525-530.

Istvan, J., \& Matarazzo, J.D. (1984). Tobacco, alcohol, and caffeine use: A review of their interrelationships. Psychological Bulletin, 95, 310-326.

Kendler, K.S., Neale, M.C., MacLean, C.L., Heath, A.C., Eaves, L.J., \& Kessler, R.C. (1993). Smoking and major depression: A causal analysis. Archives of General Psychiatry, 50, 36-43.

Marks, J.L. (1993). An evaluation of the proposed DSM-III-R criteria for the late luteal phase dysphoric disorder based on personal and family-history characteristics of women perceiving themselves as having premenstrual syndrome. Dissertation Abstracts International, 53(10), B514.

Marks, J.L., Hair, C.S., Klock, S.C., \& Ginsburg, B.E. (under review). Relationship between late luteal phase dysphoric disorder diagnosis and personal and family history of depression in women perceiving themselves to have premenstrual syndrome.

Mello, N.K. (1986). Drug use patterns and premenstrual dysphoria. In B.A. Ray \& M.C. Braude (Eds.), Women and drugs: A new era for research (DHHS Publication No. ADM87-1447). Washington, DC: U.S. Government Printing Office.

Mello, N.K., Mendelson, J.H., \& Palmieri, S.L. (1987). Cigarette smoking by women: Interactions with alcohol use. Psychopharmacology, 93, 8-15.

Mello, N.K., Mendelson, J.H., Sellers, M.L., \& Kuehnle, J.C. (1980). Effect of alcohol and marihuana on tobacco smoking. Clinical and Pharmacological Therapeutics, 27, 202-209.

Podolsky, E. (1963). Women alcoholics and premenstrual tension. Journal of the American Medical Women's Association, 18, 816-818.

Pomerleau, C.S., Cole, P.A., Lumley, M.A., Marks, J.L., \& Pomerleau, O.F. (1994). Effects of menstrual phase on nicotine, alcohol, and caffeine intake in smokers. Journal of Substance Abuse, 6 , 227-234.

Pomerleau, C.S., Garcia, A.W., Pomerleau, O.F., \& Cameron, O.G. (1992). The effects of menstrual phase and nicotine abstinence on nicotine intake and on biochemical and subjective measures in women smokers: A preliminary report. Psychoneuroendocrinology, 17, 627-638.

Pomerleau, C.S., \& Krahn, D. (1993). Smoking and eating disorders: A connection? Journal of Addictive Diseases, 12, 169.

Rivera-Tovar, A.D., \& Frank, E. (1990). Late luteal phase dysphoric disorder in young women. American Journal of Psychiatry, 147, 1634-1636.

Schechter, D., Bachmann, G.A., Vaitukaitis, J., Phillips, D., \& Saperstein, D. (1989). Perimenstrual symptoms: Time course of symptom intensity in relation to endocrinologically defined segments of the menstrual cycle. Psychosomatic Medicine, 51, 173-194.

Sommer, B. (1986). Task force report on guidelines for menstrual cycle research. Society for Menstrual Cycle Research Nezusletter, 2(3), 1-2.

Steege, J.F. (1989). Symptom measurement in premenstrual syndrome. In L.M. Demes (Ed.), Premenstrual, postpartum, and menopausal mood disorders. Baltimore, MD: Urban \& Schwarzenberg.

Steinberg, J.L., \& Cherek, D.R. (1989). Menstrual cycle and smoking behavior. Additive Behaviors, 14, 173-179.

Winer, B.J. (1971). Statistical principles in experimental design (2nd ed.). New York: McGraw-Hill. 